\title{
Surgical Approach Decision-Making Based on the CT-Guided Retrosternal Goiter Grading
}

\author{
Mohammad Reza Farahnak ${ }^{1}$, Nader Saki ${ }^{2 *}$ and Mohammad Rahmani Danalu ${ }^{3}$ \\ ${ }^{1}$ Assistant Professor or thoracic Surgery, Cancer Research center, \\ Ahvaz Jundishapur University of Medical Sciences, Ahvaz, Iran. \\ ${ }^{2}$ Associate Professor, Hearing \& Speech Research Center, \\ Ahvaz Jundishapur University of Medical Sciences, Ahvaz, Iran. \\ ${ }^{3}$ Resident of surgery, Cancer Research Center, Ahvaz \\ Jundishapur University of Medical Sciences, Ahvaz, Iran.
}

DOI: http://dx.doi.org/10.13005/bbra/1905

(Received: 16 July 2015; accepted: 23 August 2015)

\begin{abstract}
Retrosternal goiter compromises a wide range of prevalence rate in various geographical regions from 0.05 to $30.4 \%$. Retrosternal goiters produce compressive symptoms such as dyspnea and dysphagia. The Purpose of this study was to determine the type of non-cervical approach with the use of Computed Tomography (CT) findings. This study was conducted on 15 patients administered Imam Khomeini Hospital (Ahvaz, Iran) from 2009 to 2012 . From 92 clinically goiterous patients, 16 patients with extension of goiter below sternal notch in chest CT scan were enrolled. Inclusion criteria were visible thyroid tissue below the sternal notch. Patients have been studied in terms of chief complaint, age, sex, surgical approach, surgical complications and histology. Surgery begins with cervical approach and in the face of necessity non- cervical approach was used. A CSI classification system defined substernal goiter in the cranio-caudal dimension as: grade 1 (above aortic arch), grade 2 (level of aortic arch), and grade 3 (below aortic arch). We used CSI classification system for our study. CT scan should be done in three dimensional techniques with intravenous contrast. Chi-square tests are used for comparison of grading and results. P-values equal or less than 0.05 is considered statistically significant. Ten female and six male patients with a mean age of 51 years undergone surgery. Retrosternal goiter prevalence was $17 \%$. Overall, $94 \%$ patients operated by cervical and non- cervical approach, and the rest $0.06 \%$ exposed only to non- cervical approach. According to the CT findings, retrosternal goiter grades 3B and 3C need simultaneous non- cervical and cervical approaches.
\end{abstract}

Key words: Goiter, Sternotomy, Retrosternal, Computed Tomography.

Retrosternal goiter (RG) is an enlargement in thyroid gland, which presents with greater than $50 \%$ of mass below the thoracic inlet. Though the prevalence and definition of RG differ in the various sources $^{1,2}$, almost all RG are of cervical thyroid origin $^{3}$. Goiter is usually within the superioranterior mediastinum and grows to the right side; however, can extend posterior to the trachea and

\footnotetext{
* To whom all correspondence should be addressed. Tel :06132224673;

E-mail: Ahvaz.ent@gmail.com
}

esophagus ${ }^{4}$. Medical treatment of RG is not enough due to compressive symptoms and risk of malignancy, and even in asymptomatic patients require surgical intervention ${ }^{5}$. Classically RG are operated through cervical approach and in some cases non-cervical approach such as sternotomy and thoracotomy is needed ${ }^{6,7}$. Retro-esophageal goiters need to have more complex surgical intervention ${ }^{4}$. During recent years alternative medicine and non-pharmaceutical approaches for the management of different diseases have been 
significantly developed ${ }^{8-10}$. However, surgery still remains as the main option for treatment intervention in many disorders. Different classification systems based on goiter size ${ }^{7}$, location of extension ${ }^{11-13}$, relationship with the adjacent organs ${ }^{12}$, are introduced so far. Knowing the probability of need to non-cervical approach is important for surgical planning ${ }^{2}$. Therefore we attempt to make decision about the type of surgical approach based on radiological findings.

\section{MATERIALSANDMETHODS}

This study was conducted from 2009 to 2012 on 15 patients in Imam Khomeini Hospital, Ahvaz, Iran. The study was approved by Ahvaz Jundishapur University of Medical Sciences Ethical Committee, and all participants signed the informed consent prior to study. Inclusion criteria were visible thyroid tissue below the sternal notch. Patients have been studied in terms of chief complaint, age, sex, surgical approach, surgical complications and histology. Surgery begins with cervical approach and in the face of necessity noncervical approach was used. A CSI classification system defined substernal goiter in the craniocaudal dimension as: grade 1 (above aortic arch), grade 2 (level of aortic arch), and grade 3 (below aortic arch); in the anteroposterior dimension as type A (prevascular), type B (retrovascularparatracheal), and type $\mathrm{C}$ (retrotracheal); in the latero-lateral dimension as: monolateral or bilateral. We used CSI classification system for our study but want to determine which approach should be chosen for each grade and type. Computed Tomography (CT) scan should be done in three dimensional techniques with intravenous contrast. All data are statistically analyzed with spss18 software .Chi-square are used for comparison of grading and results. P-values equal or less than 0.05 was considered statistically significant.

\section{RESULTS}

The mean age of patients was 51 years. Ten patients were female (60\%) and six were male (40\%). Fifty patients came to clinic (93.3\%) with a neck mass with and without compressive signs. One patient came with mediastinal mass without a neck mass. One patient had dysphagia and two patients had dyspnea. In axial CT scanning with intravenous contrast lowest part of the goiter in relation to aortic arch (Table 1) and in sagital sections in relation to the aorta, trachea and esophagus have been identified (Table 2).

True mediastinal goiter is considered as grade 3 . Sonography reported multinodular goiter in all the patients. In 15 patients FNA study was benign and unspecified. Thoracotomy was performed in a patient with a mediastinal mass without FNA and pathology report was nodular goiter. Twelve patients operated through cervical approach three patients through cervico-sternal approach (T-shape incision) and one patient had been operated only through right thoracotomy approach. All patients underwent total

Table 1. Axial grading definition of Retrosternal Goiter

\begin{tabular}{llcc}
\hline Axial section & Definition & Number & Percentage \\
\hline Grade1 & Between sterna notch and aortic arch & 8 & $50 \%$ \\
Grade2 & At the aortic arch level & 4 & $25 \%$ \\
Grade3 & Below aortic arch & 4 & $25 \%$ \\
\hline
\end{tabular}

Table 2. Sagittal typing definition of Retrosternal Goiter

\begin{tabular}{llcc}
\hline Sagittal section & Definition & No. & Percentage \\
\hline Type A & Prevascular & 12 & 75 \\
Type B & Retrovascular,pretracheal & 1 & 6 \\
Type C & Retrotracheal & 3 & 19 \\
\hline
\end{tabular}

Abbreviation: Retrosternal Goiter (RG), Fine needle Aspiration (FNA) 
thyroidectomy and resection of the mass. Pathology of all patients was benign. In patients with T-shape incision, one patient was 3B and two patients, were 3C. Thoracotomy patient was 3C. One of the patients with T-shape incision underwent tracheostomy due to tracheomalacia. Hypocalcemia and recurrent laryngeal nerve injury were not seen.

\section{DISCUSSION}

Almost all RG patients are potentially surgical candidates because they eventually produce clinical symptoms. We used CSI classification system. Sonography, chest CT scan and FNA were part of our study but chest X-Ray has no place in our evaluations.

All type A patients including grade 1 and 2 patients operated through cervical approach. Two patients out of type C Patients operated with Tshape incision and the other operated through thoracotomy. Type B patient operated with T-shape incision. Given these findings, type A patients with grade 1 and 2 are operated through cervical approach alone. Type $\mathrm{C}$ requires non- cervical approach particularly in true mediastinal goiter thoracotomy is required. T-shape incision is advised for type B. Our net conclusion is retrosternal goiter grades $3 \mathrm{~B}$ and $3 \mathrm{C}$ need simultaneous non- cervical and cervical approaches.

\section{REFERENCES}

1. Riffat F, Del Pero MM, Fish B, Jani P. Radiologically predicting when a sternotomy may be required in the management of retrosternal goiters. Ann Otol Rhinol Laryngol. 2013;122(1):15-9.

2. Testini M, Nacchiero M, Miniello S, Ianora AS, Piccinni G, Di Venere B, et al. Management of retrosternal goiters: experience of a surgical unit.
Int Surg. 2005;90(2):61-5.

3. Armour RH. Retrosternal goitre. Br J Surg. 2000; 87(4):519.

4. Kacprzak G, Karas J, Rzechonek A, Blasiak P. Retrosternal goiter located in the mediastinum: surgical approach and operative difficulties. Interact Cardiovasc Thorac Surg. 2012; 15(5): 935-7.

5. Tsakiridis K, Visouli AN, Zarogoulidis P, Karapantzos E, Mpakas A, Machairiotis N, et al. Resection of a giant bilateral retrovascular intrathoracic goiter causing severe upper airway obstruction, 2 years after subtotal thyroidectomy: a case report and review of the literature. J Thorac Dis. 2012; 4(Suppl 1):41-8.

6. Gao B, Jiang Y, Zhang X, Zhao J, He Y, Wen Y, et al. Surgical treatment of large substernal thyroid goiter: analysis of 12 patients. Int J Clin Exp Med. 2013; 6(7): 488-96.

7. Rugiu MG, Piemonte M. Surgical approach to retrosternal goitre: do we still need sternotomy? Acta Otorhinolaryngol Ital. 2009; 29(6):331-8.

8 Yadollahpour A, Jalilifar M. Electromagnetic Fields in the Treatment of Wound: A Review of Current Techniques and Future Perspective. $J$ Pure Appl Microbio. 2014; 8(4): 2863-77.

9. Yadollahpour A, Rezaee Z. Electroporation as a New Cancer Treatment Technique: A Review on the Mechanisms of Action. Biomedical \& Pharmacology Journal. 2014;7(1):53-62.

10. Zohre R, Ali Y, Mostafa J, Samaneh R. Nondrug Antimicrobial Techniques: Electromagnetic Fields and Photodynamic Therapy. Biomedical \& Pharmacology Journal. 2015; 8(March Spl Edn.): 147-55.

11. Ignjatovic M, Stanic V, Cuk V, Kostic Z. Intrathoracic goiter: analysis of 21 cases. Acta Chir Iugosl. 2002; 49(1): 15-25.

12. Huins CT, Georgalas C, Mehrzad H, Tolley NS. A new classification system for retrosternal goitre based on a systematic review of its complications and management. Int J Surg. 2008; 6(1):71-6.

13. Seyyed Abdolhossein Masoumi, Amir Fasihi, Nader Nazari, Soheila Nikakhlagh. Incidence of thyroid papillary cancer in contralateral lobe. Apadana Journal of Clinical Research 2013; 1:17-22 